Rabaska

Revue d'ethnologie de l'Amérique française

\title{
Jean de paroles
}

\section{Philippe Dubé}

Volume 5, 2007

URI : https://id.erudit.org/iderudit/019036ar

DOI : https://doi.org/10.7202/019036ar

Aller au sommaire du numéro

Éditeur(s)

Société québécoise d'ethnologie

ISSN

1703-7433 (imprimé)

1916-7350 (numérique)

Découvrir la revue

Citer ce document

Dubé, P. (2007). Jean de paroles. Rabaska, 5, 122-125.

https://doi.org/10.7202/019036ar

Ce document est protégé par la loi sur le droit d'auteur. L'utilisation des services d'Érudit (y compris la reproduction) est assujettie à sa politique d'utilisation que vous pouvez consulter en ligne.

https://apropos.erudit.org/fr/usagers/politique-dutilisation/
Cet article est diffusé et préservé par Érudit.

Érudit est un consortium interuniversitaire sans but lucratif composé de l'Université de Montréal, l'Université Laval et l'Université du Québec à Montréal. Il a pour mission la promotion et la valorisation de la recherche. https://www.erudit.org/fr/ 


\title{
Jean de paroles
}

\author{
PhilipPe Dubé \\ Université Laval, Québec
}

Je tiens d'abord à remercier Jean Simard qui m'a demandé de réagir à un texte que j'attendais depuis longtemps : celui de Jean Du Berger. Je dois dire que je me sens très honoré de participer à cette table ronde, assis entre vous deux, vous qui avez été mes maîtres en quelque sorte, on pourrait dire mes idoles, afin de commenter et de tenter de nourrir une réflexion sur un texte qui est déjà très riche.

Dans un premier temps, je dirais que l'article de Jean Du Berger a le grand avantage de mettre la table et de bien situer ce qu'est la pratique orale, plus particulièrement le conte, du point de vue ethnologique. D'entrée de jeu, il aborde la question de la pratique orale d'une manière très fine et nous amène progressivement sur le terrain de la performance. C'est d'ailleurs un mot qui m'a beaucoup fait réagir parce qu'il y a en effet dans la pratique orale une dimension qui n'est jamais retenue par les technologies de l'enregistrement et que, de ce point de vue, - je reprends ses termes - il y a un « ici-et-maintenant » qui n'est plus présent dans l'acte même de l'archivage de ce matériel évidemment unique. Il décrit au fond ce qu'est, non pas la littérature orale au sens large, mais - une idée encore que j'aime bien - la pratique orale, la pratique narrative comme il dit. Et il nous amène à cette idée extrêmement riche en posant le problème de la transférabilité - la « traduction » dirait Ricœur - d'un témoignage retenu par des moyens techniques et qui nous livre une partie seulement de ce qui a été dit, car il y a tout un contexte qui nécessairement n'est plus présent, n'est pas contenu par l'intermédiaire - en fait par le médium qui recueille cette matière. Et c'est là, je pense, tout le problème de fond : c'est dans un premier temps un problème de médium, parce que, comme on le dit, la parole est volatile; et le fait de donner à cette parole un statut de pérennité, de confronter en somme la parole au musée, est quelque chose d'extrêmement hasardeux. Et Jean Du Berger pose parfaitement bien la question : « Le lieu que constitue un musée apparaitil comme correspondant aux attentes d'un tel projet ? » Dit autrement, est-ce que le musée a la compétence nécessaire pour retenir, non seulement cette parole - parce que la parole est déjà retenue par l'archive et que le musée lui 
devrait aller plus loin, et c'est là tout le problème -, mais pour enfin dépasser le simple mode d'archivage de la conservation, qui est déjà réglé par les archives de folklore ? On a en fait une banque énorme de documents. Le problème est bien évidemment alors de savoir qu'est-ce qu'on fait de ces documents et, ensuite, de se demander si ces documents tels qu'ils sont consignés sont transmissibles.

Dans ce même texte, Jean Du Berger traite de l'importance de la pratique orale, non seulement en tant que performance (tout le non-dit de la pratique narrative), mais il rappelle aussi qu'il y a des conditions qui doivent être réunies pour donner de la valeur à ce qu'on pourrait appeler un « logofact » peut-être. Et le groupe d'appartenance est, entre autres choses, une de ces conditions. Il n'y a pas de parole réelle en fait qui mérite, même à la limite, d'être retenue si elle n'est pas ancrée, associée directement à un groupe et au lieu au sens plein du terme qui a permis cette parole. - Dans son cours d'ethnologie urbaine, je me souviens bien, Jean Du Berger proposait de transformer le magasin Miracle Mart à Sainte-Foy en musée. Je revis ce temps-là où il nous invitait, au delà des légendes à faire revivre, à imaginer un lieu capable d'accueillir un patrimoine vivant dans la ville, et de pouvoir porter ces paroles urbaines. - En lisant son texte aujourd'hui, on découvre toute la complexité du projet qui doit réunir, non seulement le groupe d'appartenance, non seulement le lieu, non seulement la parole qui est énoncée, mais aussi la personnalité du conteur. Et tous ces éléments-là sont évacués parce qu'ils n'ont pas été retenus par le travail trop spécifique de l'archivage. Et c'est là tout le problème de trouver un lieu qui pourrait à la fois rendre compte de cette complexité qui est contenue dans la parole.

Alors le musée est-il incompétent pour autant ? Il pose la question, à mon sens, à deux chapitres. Et c'est là que le muséologue peut tenter de vous éclairer. Le premier élément, c'est qu'on demande au musée de faire durer l'éphémère et non pas seulement de capter l'essentiel. Dans la nature même de la parole, il y a quelque chose d'éphémère. Il y a une sorte de contradiction évidente à vouloir faire durer cet éphémère. Cette parole a la qualité qu'elle a parce qu'elle est éphémère justement ; elle est prise dans un moment donné, dans un contexte donné avec des personnes précises, et ceci n'est pas reproductible, ou en tout cas, c'est difficilement traduisible. Alors revenons à la question de départ, est-ce que le musée est compétent pour faire durer l'éphémère ? À bien considérer ce que fait le musée - ici le musée de société où le musée se définit plutôt comme un lieu de mémoire, un lieu qui tente de faire durer ce qui dure -, c'est un problème qui n'est pas simple à résoudre.

Le deuxième élément - qui empêche le musée d'être compétent à porter d'une manière intéressante, divertissante, interactive, participative, cette 
parole - est la difficulté pour le musée de pouvoir recréer les conditions préalables à la pratique narrative. Comment le musée peut-il arriver à recréer ce qu'évoquait Félix-Antoine Savard, cette idée d'être sous un arbre à une heure donnée de la journée, où il y a de la magie qui nécessairement n'a pas été consignée en fait au moment de la cueillette ? Et là, on demande au musée d'aller au-delà de ça et de créer ces conditions préalables à la pratique narrative.

Alors, pour répondre à cette question, qui est double - faire durer l'éphémère dans un premier temps et, dans un deuxième temps, tenter de créer des conditions qui permettent la pratique narrative-, je ferais une réponse double aussi. En me référant à la question de Jean Du Berger, le lieu que constitue le musée peut, à mon sens, répondre à de telles attentes et je dirais oui dans un premier temps. Mais d'autre part la réponse pourrait aussi être non. J'ai cherché un modèle de musée qui pourrait éventuellement être mis en rapport avec le conte, car il n'a pas l'exclusivité de la pratique narrative. J'ai pensé à l'humour, notamment au musée Juste pour rire qui consigne en fait une parole qui est dite. Il y a là aussi un contexte, un groupe d'appartenance, une personnalité. Puis, je me suis amusé, sur la toile, à me refamiliariser avec ce lieu. Pour tout dire, c'est vraiment de l'anti-humour, de l'anti-pratique narrative; on est en fait très loin de l'objectif. Quand on consulte ne serait-ce que l'album de photographies que nous propose le musée virtuel de l'humour, le musée Juste pour rire, c'est d'une tristesse : parce que ce ne sont que des images qui défilent comme ça tout bonnement. Il n'y a plus rien de vrai, d'authentique ; il n'y a plus cette magie de l'humour, cette magie de la pratique narrative qui porte l'humour et qui fait le véritable succès du festival de l'humour pour le nommer. Et qu'on en soit ou pas, il reste que c'est un phénomène de société qui est important et dont on doit rendre compte. Et le musée le fait, mais à mon sens, d'une manière qui est très loin du projet du Miracle Mart transformé de Jean Du Berger. On est dans un lieu de consignation qui est d'ordre artéfactuel, car on se darde sur l'objet qui vient témoigner de cette parole, mais on est loin de la parole elle-même. Et c'est là tout le problème du musée Juste pour rire.

Par contre - et ce n'est pas pour flatter mon collègue Benoît Gauthier -, mais quand je regarde d'un peu plus près le Musée québécois de culture populaire, que j'ai visité l'automne dernier, j'ai été littéralement séduit par une de ses expositions parce qu'elle témoignait justement de l'histoire d'un lieu à travers la parole. Ce qui m'a le plus intéressé dans cette exposition, c'est la qualité des témoignages des gens du quartier qui nous sont livrés en film, une parole animée, évidemment décontextualisée, mais en même temps qui est portée par la personne que l'on peut au moins voir. On a fait là un pas 
de plus que les archives ; en plus d'entendre, on voit la personne qui s'exprime et qui, finalement, vit sa parole. Et je me dis qu'ainsi on s'approche peut-être du musée de Jean Du Berger, du musée de l'oral qui a évidemment à être défini d'une manière plus large.

Ce n'est pas que le texte ne structure pas assez le projet. Le texte lance des idées et je pense que Jean Du Berger le dit très bien : «Par le conte, les cultures se rencontrent ». Il y a là tout un programme qui se dégage d'un pareil musée où finalement la parole serait le centre du musée lui-même. Mais c'est évident que la parole seule, on le voit très bien à travers les archives, ne peut pas porter le projet d'un musée. Et je vous demanderais simplement de réfléchir sur la question. Au bout du compte, j'aurais plutôt tendance à abandonner l'idée de musée. Avec ma double réponse, affirmative et négative, le musée n'a plus tout à fait l'ampleur du projet de Jean Du Berger. C'est pourquoi j'opterais tout bonnement pour la Maison du conte. Vous allez me dire que je joue un peu avec les mots, peut-être mais, à mon sens, une maison, que ce soit une maison d'écriture ou de la parole, permet davantage de réaliser le projet qui justement ne veut pas écarter la parole du lieu qui l'a vue naître, des personnes qui la disent et du moment où elle est livrée. Et, de ce point de vue-là, je pense que le musée qui est revenu en force au XVII siècle, au moment même où on a créé la guillotine - c'est Georges Bataille qui met en relation le musée avec la guillotine : un lieu qui coupe, un lieu qui tue, un lieu qui met à mort, il ne faut pas l'oublier -, le musée est fondamentalement un lieu de mort, même si on fait tout pour le faire vivre. Il reste que, si on veut faire place à la parole entière et totale, comme Jean Du Berger nous le suggère, c'est à mon sens dans une Maison (la maison de la mémoire est un concept belge) qu'on trouvera le lieu idéal pour porter cette parole, pour la faire vivre et surtout pour inviter tous ceux qui ont le goût de dire les choses. Pour sortir du rapport assez restreint qu'on a avec cette parole-là, pour recréer cet « iciet-maintenant », ce moment magique, j'ai l'impression qu'une grande Maison du conte serait ce lieu tant attendu. Ce projet m'apparaît davantage porteur que de le concevoir dès le point de départ comme un musée et encore moins comme un Miracle Mart de la parole. 